\title{
DIMENSION REDUCTION IN HETEROGENEOUS NEURAL NETWORKS: GENERALIZED POLYNOMIAL CHAOS (GPC) AND ANALYSIS-OF-VARIANCE (ANOVA)
}

\author{
MINSEOK CHOI, TOM BERTALAN, CARLO R. LAING, AND IOANNIS G KEVREKIDIS
}

\begin{abstract}
We propose, and illustrate via a neural network example, two different approaches to coarse-graining large heterogeneous networks. Both approaches are inspired from, and use tools developed in, methods for uncertainty quantification in systems with multiple uncertain parameters - in our case, the parameters are heterogeneously distributed on the network nodes. The approach shows promise in accelerating large scale network simulations as well as coarse-grained fixed point, periodic solution and stability analysis. We also demonstrate that the approach can successfully deal with structural as well as intrinsic heterogeneities.
\end{abstract}

\section{INTRODUCTION}

Systems of coupled identical oscillators can often be studied exploiting this special symmetry (invariance to permuting their identities [1); yet most realistic systems possess some form/degree of heterogeneity, and thus studying the influence of this heterogeneity on dynamics is of crucial importance. While for a small number of oscillators the dynamics of each and every one can be easily simulated, for larger networks this becomes impractical, particularly if one is interested in typical behaviour of similar networks, not just the behavior of a single, particular network realization. Thus techniques for dimension reduction, i.e. faithfully representation of a heterogeneous network by a lower-dimensional dynamical system, are useful in the dynamic/parametric study of such networks.

In this paper we demonstrate the use of two such dimensionality reduction techniques for different heterogeneous networks of coupled model neurons. The first network we consider is all-to-all coupled, but four of the physiological parameters associated with the dynamical mechanisms occurring within each neuron are heterogeneous. The term that embodies all-to-all coupling of the neurons is then approximated by a four-dimensional integral over these heterogeneous parameters. We approximate this integral using the ANalysis-Of-VAriance (ANOVA) method and expand the instantaneous states of the neurons in polynomials in the four heterogeneous parameters. A small number of time-dependent coefficients for these polynomials constitute the variables of a reduced model for the network. We demonstrate the computational efficiency of this reduction with several computations within the equation-free framework (e.g. [14, 23]).

The second network we consider is both intrinsically heterogeneous (a physiological parameter associated with the individual neuron dynamics is different for each neuron) as well as structurally heterogeneous (because the neurons are connected in a nontrivial way). We observe that the state of each neuron can be accurately expressed as a sum of polynomials in both the intrinsic heterogeneity parameter and a neuron's degree (number of connections) in the network. This is a generalized Polynomial Chaos (gPC) approach, and the polynomials are orthogonal with respect to a density that depends on the probability distribution of the heterogeneous intrinsic parameter as well as the 
degree distribution of the network. A small number of the coefficients of these polynomials again helps construct an accurate reduced model of the network dynamics.

The model is presented in Sec. 2 and we then briefly review both ANOVA and the gPC method. Numerical examples are given in Sec. 3 and we conclude with a discussion in Sec. 4 .

\section{THE MODEL}

We consider a network of model neurons previously studied as a model for rhythmic oscillations in the pre-Bötzinger complex [17, 20]:

$$
\begin{aligned}
C \frac{d V_{i}}{d t} & =-g_{N a} m\left(V_{i}\right) h_{i}\left(V_{i}-V_{N a}\right)-g_{l}\left(V_{i}-V_{l}\right)+I_{s y n}^{i}+I_{a p p}^{i}, \\
\frac{d h_{i}}{d t} & =\frac{h_{\infty}\left(V_{i}\right)-h_{i}}{\tau\left(V_{i}\right)}
\end{aligned}
$$

for $i=1, \ldots, N$, where

$$
I_{\text {syn }}^{i}=\frac{g_{\text {syn }}\left(V_{\text {syn }}-V_{i}\right)}{N} \sum_{j=1}^{N} A_{i, j} s\left(V_{j}\right) .
$$

Here $V_{i}$ is the membrane potential of neuron $i$, and $h_{i}$ is a channel state variable for neuron $i$ that governs the inactivation of persistent sodium. The first and second term of the right hand side in Equation (1a) is a persistent sodium current and passive leakage current, respectively and $g_{N a}, V_{N a}, g_{l}, V_{l}$ are corresponding nominal parameters [3, 4]. Equation (1) was derived from the models in Butera et al. [3, 4, by blocking currents responsible for action potentials; Rubin [21] considered a similar model with $N=2$, and Dunmyre and Rubin $[5]$ considered synchronization in the case $N=3$. The various functions involved in the model equations are as follows:

$$
\begin{aligned}
s(V) & =\frac{1}{1+\exp (-(V+40) / 5)}, \\
\tau(V) & =\frac{1}{\epsilon \cosh ((V+44) / 12)}, \\
h_{\infty}(V) & =\frac{1}{1+\exp ((V+44) / 6)}, \\
m(V) & =\frac{1}{1+\exp (-(V+37) / 6)} .
\end{aligned}
$$

The functions $\tau(V), h_{\infty}(V)$ and $m(V)$ are a standard part of the Hodgkin-Huxley formalism [12, and synaptic communication is assumed to act instantaneously through the function $s(V)$. The neurons are coupled through a synaptic current $I_{s y n}^{i}$ for $g_{s y n} \neq 0$ where $A_{i j}$ is a symmetric adjacency matrix, i.e. $A_{i j}=1$ if neuron $i$ and $j$ are connected, and $A_{i j}=0$ otherwise. A previous study considered only all-to-all coupled networks [17, but we will consider a more structured network in Sec. 3.2 below. We denote the degree of $i$-th neuron (its number of neighbors) by $\kappa_{i}$, i.e. $\kappa_{i}=\sum_{j \neq i}^{N} A_{i j}$.

It was shown in [17, 20] that if the values of the applied currents $I_{a p p}^{i}$ are uniformly distributed in a certain interval, synchronous behavior is observed after a transient, i.e. all neurons oscillate periodically with the same period, although the heterogeneity in the $I_{a p p}^{i}$ means that each neuron follows a slightly different periodic orbit in its own $(V, h)$ phase space. It appears that (asymptotically in time) the values of the $V_{i}$ and $h_{i}$ vary smoothly as a function of the heterogeneous 
parameter $I_{a p p}^{i}$. This observation lead to the continuum limit of Equations (11):

$$
\begin{aligned}
C \frac{\partial V(\mu, t)}{\partial t} & =-g_{N_{a}} m(V(\mu, t)) h(\mu, t)\left(V(\mu, t)-V_{N_{a}}\right)-g_{l}\left(V(\mu, t)-V_{l}\right)+I_{\text {syn }}+I_{a p p} \\
\frac{\partial h(\mu, t)}{\partial t} & =\frac{h_{\infty}(V(\mu, t))-h(\mu, t)}{\tau(V(\mu, t))}
\end{aligned}
$$

where $I_{a p p}$ is parameterized as $I_{a p p}=I_{m}+I_{s} \mu$ with $\mu$ being a uniform distribution on $[-1,1]$, i.e. $I_{a p p}$ follows a uniform distribution on $\left[I_{m}-I_{s}, I_{m}+I_{s}\right]$ and

$$
I_{\text {syn }}(\mu, t)=g_{\text {syn }}\left(V_{\text {syn }}-V(\mu, t)\right) \int_{-1}^{1} s(V(\mu, t)) p(\mu) d \mu .
$$

Note that $p(\mu)$ is a probability density function for $\mu$, i.e. $p(\mu)=1 / 2$ for $-1 \leq \mu \leq 1$. In this limit $V_{i}(t)$ and $h_{i}(t)$ become the functions $V(\mu, t)$ and $h(\mu, t)$, respectively. The results for $N \rightarrow \infty$ should provide a good approximation to the behavior seen when $N$ is large but finite, as we expect it to be. Rubin and Terman 20 first introduced the continuum limit, their contribution being largely analytical. Laing et al. [17 presented a computationally efficient way to describe the heterogeneous network by applying techniques widely used in the uncertainty quantification (UQ) community known as generalized Polynomial Chaos and the associated stochastic collocation method (SCM) [26, 27, 11]. These methods are high-order accurate, in fact exponentially accurate, but suffer when the dimensionality of the parametric space increases; this is known as the so-called curse of dimensionality. Sparse grids techniques have greatly alleviated this problem by utilizing the smoothness of the function in low to moderate "heterogeneity dimensions" [7, 10]. However, the complexity estimate of sparse grids still depends heavily on the dimension and on the regularity of the functions being integrated. To push the dimensionality barrier higher, several methods have been introduced in the UQ commnunity; one of them is the ANOVA method, which will be described in Sec. 3.1 for a case in which there are multiple heterogeneous physiological (intrinsic to each neuron) parameters. In the subsections 2.1 and 2.2 we briefly review the gPC and ANOVA methods; see [7, 27, 28, 11] for more details.

2.1. Polynomial Chaos as a low-dimensional representation. The Polynomial Chaos (PC) method is widely used in the UQ community [27, 11. The method has also been applied successfully to coarse-graining the dynamics of heterogeneous networks, for which some parameters intrinsic to each neuron are distributed in a prescribed way [17, 19]. The PC expansion involves representing the state variable $\boldsymbol{X}=\left(x_{1}, \ldots, x_{n}\right)$ as a weighted series of orthogonal basis functions (polynomials) of the heterogeneous parameters $\boldsymbol{\xi}=\left(\xi_{1}, \ldots \xi_{m}\right)$ :

$$
\boldsymbol{X}(t ; \boldsymbol{\xi})=\sum_{i} \boldsymbol{\alpha}_{i}(t) \boldsymbol{\Psi}_{i}(\boldsymbol{\xi})
$$

where $\boldsymbol{\Psi}_{i}(\boldsymbol{\xi})$ is the $i$-th basis function and the $\boldsymbol{\alpha}_{i}(t)$ are PC coefficients. Conversely, the coefficients $\boldsymbol{\alpha}_{i}$ can be recovered by the projection on the basis $\Psi_{i}(\boldsymbol{\xi})$ due to the orthonormality of the basis functions

$$
\boldsymbol{\alpha}_{i}=\left\langle\boldsymbol{X}, \boldsymbol{\Psi}_{i}\right\rangle \equiv \int X(\boldsymbol{\xi}) \Psi_{i}(\boldsymbol{\xi}) d P(\boldsymbol{\xi})
$$

where the inner product $\langle\cdot, \cdot\rangle$ is defined by integration with respect to the underlying measure $d P(\boldsymbol{\xi})$.

Assuming independence of the distributions of the heterogeneous parameters, $\boldsymbol{\Psi}_{i}$ can be separated into a tensor product of independent scalar polynomial bases $\boldsymbol{\Psi}_{i}(\boldsymbol{\xi})=\prod_{k=1}^{m} \Psi_{i_{k}}\left(\xi_{k}\right)$. For 
well-known distributions such as uniform or normal, there are corresponding PC basis functions: Legendre or Hermite polynomial, respectively. In the case of arbitrarily distributed heterogeneous parameters, a PC basis can be constructed numerically 24. With a basis chosen, the system of coupled ODEs for $\boldsymbol{X}$

$$
\frac{d \boldsymbol{X}(t ; \omega)}{d t}=\boldsymbol{f}(\boldsymbol{X} ; \boldsymbol{\xi})
$$

(of which (11) is a specific example) can be recast as a system of ODEs for the PC coefficients $\boldsymbol{\alpha}_{i}$ via the Galerkin method

$$
\frac{d \boldsymbol{\alpha}_{j}}{d t}=\left\langle\boldsymbol{f}\left(\sum_{i} \boldsymbol{\alpha}_{i} \boldsymbol{\Psi}_{i}\right), \boldsymbol{\Psi}_{j}\right\rangle,
$$

where the orthogonality of the basis functions is exploited.

A computational task involving simulating each neuron in a system such as Equation (1) is too complicated if the number of neurons is large, and hence an accurate coarse-grained description is useful (if it exists). It turns out that PC coefficients $\boldsymbol{\alpha}_{i}$ serve well as coarse-grained descriptors of a system like (11) with heterogeneous parameters [17, 19. Note that the number of coefficients is usually much less than the number of variables in Equation (11). This model reduction, as we will show below, allows us to perform a number of coarse-grained modeling tasks such as accelerated simulation via Coarse Projective Integration (CPI) or accelerated limit cycle computation, accompanied by coarse-grained stability analysis [2, 15, 17, 18, 19].

Coarse-graining this model requires the computation of two high-dimensional integrals-the coupling integral (8), and the inner product (10). To this end, we introduce the ANOVA method in the following subsection.

2.2. ANOVA. ANOVA is widely used as a statistical method to test differences between two or more means [13, 6. The same idea can be used for the interpolation and integration of high dimensional problems as well as analyzing stochastic simulations. 8, 22. Consider an integrable function $f(\boldsymbol{x}), \boldsymbol{x}=\left(x_{1}, x_{2}, \cdots, x_{N}\right)$ defined in $I^{N}=[0,1]^{N}$. The ANOVA representation for $f(\boldsymbol{x})$ is as follows:

Definition 1. The representation of $f(\boldsymbol{x})$ in a form

$$
f(\boldsymbol{x})=f_{0}+\sum_{s=1}^{N} \sum_{j_{1}<\cdots<j_{s}} f_{j_{1} \cdots j_{s}}\left(x_{j_{1}}, \cdots, x_{j_{s}}\right)
$$

or equivalently

$$
f(\boldsymbol{x})=f_{0}+\sum_{1 \leq j_{1} \leq N} f_{j_{1}}\left(x_{j_{1}}\right)+\sum_{1 \leq j_{1}<j_{2} \leq N} f_{j_{1}, j_{2}}\left(x_{j_{1}}, x_{j_{2}}\right)+\cdots+f_{1,2, \cdots, N}\left(x_{1}, x_{2}, \cdots, x_{N}\right)
$$

is called the ANOVA decomposition of $f(\boldsymbol{x})$, if

$$
f_{0}=\int_{I^{N}} f(\boldsymbol{x}) \mathrm{d} \mu(\boldsymbol{x})
$$

and

$$
\int_{I} f_{j_{1} \cdots j_{s}} \mathrm{~d} \mu\left(x_{j_{k}}\right)=0 \quad \text { for } \quad 1 \leq k \leq s
$$

We call $f_{j_{1}}\left(x_{j_{1}}\right)$ the first-order term (or first-order component function), $f_{j_{1}, j_{2}}\left(x_{j_{1}, j_{2}}\right)$ the secondorder term (or second-order component function), etc. 
The terms in the ANOVA decomposition are computed as follows:

$$
f_{S}=\int_{I^{N-|S|}} f(\boldsymbol{x}) \mathrm{d} \mu\left(\boldsymbol{x}_{S^{c}}\right)-\sum_{T \subset S} f_{T}\left(\boldsymbol{x}_{T}\right),
$$

where $S=\left\{j_{1}, j_{2}, \cdots, j_{s}\right\},|S|$ is the number of elements in $S, T=\left\{i_{1}, i_{2}, \cdots, i_{t}\right\}$ is a subset of $S$, i.e. $\left\{i_{1}, i_{2}, \cdots, i_{t}\right\} \subset\left\{j_{1}, j_{2}, \cdots, j_{s}\right\}$ and $f_{T}=f_{i_{1}, i_{2}, \cdots, i_{t}}\left(x_{i_{1}}, x_{i_{2}}, \cdots, x_{i_{t}}\right)$.

An important property of the ANOVA decomposition of $f$ is that the variance of $f$ is the sum of the variances of all the ANOVA terms except $f_{0}$ :

$$
\sigma^{2}(f)=\sum_{s=1}^{N} \sum_{|S|=s} \sigma^{2}\left(f_{S}\right), \quad \sigma^{2}\left(f_{S}\right)=\int_{I^{N}} f_{S}^{2} d \mu(x),
$$

or equivalently:

$$
\sigma^{2}(f)=\sum_{1 \leq j_{1} \leq N} \sigma^{2}\left(f_{j_{1}}\right)+\sum_{1 \leq j_{1}<j_{2} \leq N} \sigma^{2}\left(f_{j_{1}, j_{2}}\right)+\cdots+\sigma^{2}\left(f_{1,2, \cdots, N}\right)
$$

Computing the ANOVA decomposition, i.e. the constant term and high-order terms from Equations (15) and (17) respectively, can be very expensive for high dimensional problems or complicated functions $f(\boldsymbol{x})$. One therefore uses the Dirac measure instead of the Lebesgue measure in integrations, i.e., $\mathrm{d} \mu(\boldsymbol{x})=\delta(\boldsymbol{x}-\boldsymbol{c}) \mathrm{d} \boldsymbol{x}, \boldsymbol{c} \in I^{N}$. The point " $\boldsymbol{c}$ " is called the "anchor point" and this method is called "anchored-ANOVA". Then the (approximate) evaluation of the integral that appears in the first term of the right hand side of Equation (17) becomes much easier. For example, for the constant term and first-order term we have

$$
\begin{aligned}
f_{0} & =f(\boldsymbol{c}) \\
f_{j}\left(x_{j}\right) & =f\left(c_{1}, \ldots, c_{j-1}, x_{j}, c_{j+1}, \ldots, c_{N}\right)-f_{0}, \quad j=1, \ldots, N .
\end{aligned}
$$

Note also that Equation (17) implies that the $|S|$-order terms can be constructed recursively from all ANOVA terms whose orders are less than $|S|$.

For numerical purposes we approximate $f(\boldsymbol{x})$ by all ANOVA terms whose degrees are less than or equal to $\nu$ :

$$
f(\boldsymbol{x}) \approx f_{0}+\sum_{j_{1} \leq N} f_{j_{1}}\left(x_{j_{1}}\right)+\sum_{j_{1}<j_{2} \leq N} f_{j_{1}, j_{2}}\left(x_{j_{1}}, x_{j_{2}}\right)+\cdots+\sum_{j_{1}<j_{2}<\cdots<j_{\nu} \leq N} f_{j_{1}, j_{2}, \cdots, j_{\nu}}\left(x_{j_{1}}, x_{j_{2}}, \cdots x_{j_{\nu}}\right) .
$$

Here $N$ is called nominal dimension, and $\nu$ is called the truncation or effective dimension. If $\nu$ is low, then this type of approach, i.e. approximating the $N$-dimensional problem into a series of lower-dimensional problem, can greatly alleviate the computational burden. For example, let us consider the integration of the function $\int f(\boldsymbol{x}) d \boldsymbol{x}$, e.g., $f$ here can be the integrand in Equation (8) or Equation (10). Since the integration is a linear operator, the integral can be approximated by the sum of integrals of ANOVA terms, i.e.

$$
\int_{I^{N}} f(\boldsymbol{x}) d \boldsymbol{x} \approx \int_{I^{N}} f_{0} d \boldsymbol{x}+\sum_{s=1}^{\nu} \sum_{j_{1}<\cdots<j_{s}} \int_{I^{N}} f_{j_{1} \cdots j_{s}}\left(x_{j_{1}}, \ldots, x_{j_{s}}\right) d \boldsymbol{x} .
$$

Then, the N-dimensional integration problem becomes much lower dimensional (up to $\nu$ assuming $\nu \ll N)$ integration, where we can use collocation methods such as those involving Gaussian quadrature and weights. Consider the first-order term $f_{1}\left(x_{1}\right)$ for instance. Let $\boldsymbol{c}_{-1}=\left(c_{2}, c_{3}, \ldots, c_{N}\right)$ and $\left(q_{1}^{j}, w^{j}\right)_{j=1}^{\mu}$ be the quadrature points and corresponding weights for integration along the first 
dimension, with $\mu$ being the number of quadrature points. Then, the integration of $f_{1}\left(x_{1}\right)$ can be approximated by

$$
\int_{I^{N}} f_{1}\left(x_{1}\right) d \boldsymbol{x} \approx \sum_{j=1}^{\mu} f_{1}\left(q_{1}^{j}\right) w^{j}=\sum_{j=1}^{\mu}\left(f\left(q_{1}^{j}, c_{2}, c_{3}, \ldots, c_{N}\right)-f_{0}\right) w^{j} .
$$

See [8, 28, for more details. In [28, the authors applied the ANOVA method for a stochastic incompressible flow problem with a nominal dimension of parametric space up to 100 but with an effective dimension of 2 as an efficient dimension-reduction technique. In Sec. 3.1 below we will demonstrate the use of ANOVA to approximately describe coupled neuronal networks that have multiple independent heterogeneous parameters.

\section{NUMERICAL EXAMPLES}

In this section, two cases are presented to illustrate the gPC and ANOVA methods to model the effect of multiple heterogeneous parameters. In the first case we model four distinct heterogeneous parameters in order to demonstrate the ANOVA method: $I_{a p p}, g_{N_{a}}, V_{s y n}$ and $V_{N_{a}}$ are all assumed to be uniformly distributed. For simplicity we do not assume structural heterogeneity, i.e. neurons are all-to-all coupled yielding $A_{i j}=1$ for all $i, j$; the case of simultaneous intrinsic and strctural heterogeneity will be discussed next. After comparing the ANOVA method with sparse grids or the "direct" Monte Carlo (MC) method, we perform a number of coarse-grained modeling tasks such as Coarse Projective Integration and coarse-grained stability analysis.

We then consider the network of neurons to be heterogeneous in the following sense: neuron $i$ has an applied current $I_{a p p}^{i}$, which is referred to as an intrinsic heterogeneity, and a degree $\kappa_{i}$, which is referred to as a structural heterogeneity. (The $I_{a p p}^{i}$ are not all equal, and neither are the $\kappa_{i}$.) The results suggest that the techniques used here may be also applicable to this type of network.

3.1. Case I: Multiple heterogeneous parameters. We consider the case where there exist four heterogeneous parameters: $I_{a p p}, g_{N_{a}}, V_{s y n}$ and $V_{N_{a}}$ are all independently and uniformly distributed. Each of these four parameters can be parameterized by their mean and half-width, together with the standard uniform distribution $\xi_{i}, i=1,2,3,4$, which we denote by $\xi_{i} \sim U[-1,1]$, and whose

probability distribution function is $p\left(\xi_{i}\right)=\frac{1}{2}$ for $-1 \leq \xi_{i} \leq 1$. For example, if $I_{a p p} \sim U[17.5,32.5]$, then it is parameterized as $I_{a p p}=E\left[I_{a p p}\right]+h\left(I_{a p p}\right) \xi$ where $E\left[I_{a p p}\right]=25$ and $h\left(I_{a p p}\right)=7.5$ are the mean and half-width of $I_{a p p}$, respectively, and $\xi$ is the standard uniform distribution. Then, as mentioned in the above section, the continuous variables $V$ and $h$ become a function of these $\xi_{i}$ 's as well as time $t$ as $V\left(t ; \xi_{1}, \xi_{2}, \xi_{3}, \xi_{4}\right)$ and $h\left(t ; \xi_{1}, \xi_{2}, \xi_{3}, \xi_{4}\right)$, respectively and the sum in Equation (2) is represented by the integral

$$
\int_{\Omega^{4}} s(V(t ; \boldsymbol{\xi})) p(\boldsymbol{\xi}) \mathrm{d} \xi_{i}
$$

where $\boldsymbol{\xi}=\left(\xi_{1}, \xi_{2}, \xi_{3}, \xi_{4}\right), p(\boldsymbol{\xi})=\prod_{i=1}^{4} p_{i}\left(\xi_{i}\right)$ and $\Omega=[-1,1]$. In stochastic collocation or sparse grid methods this integral is approximated as the sum of the function evaluated at the collocation points multiplied by their corresponding weights; see [17, 26] for more detail. In ANOVA methods, we first approximate the function $s(V(t ; \vec{\xi}))$ by its ANOVA terms whose orders are less than $\nu$ as in Equation (21). Then the integral of a high-dimensional function is represented by the integral of a series of low-order functions, which can be easily computed by standard numerical integration 
techniques. For example, assume that $\nu=1$. Then the ANOVA approximation of $s$ denoted by $s_{A}$ is as follows:

$$
s(V(t ; \boldsymbol{\xi})) \approx s_{A}(V(t ; \boldsymbol{\xi}))=s_{0}+\sum_{j=1}^{4} s_{j}\left(\xi_{j}\right)
$$

where $s_{j}\left(\xi_{j}\right)$ is given in Equation (20). For example, for $j=2, s_{2}\left(\xi_{2}\right)=s\left(V\left(t ; c_{1}, \xi_{2}, c_{3}, c_{4}\right)\right)$ for an anchor point $\boldsymbol{c}=\left(c_{1}, c_{2}, c_{3}, c_{4}\right)$. Then the integral in Equation (24) is computed as the sum of the integral of the constant term and the first-order ANOVA terms, which are readily computable:

$$
\int_{\Omega^{4}} s(V(t ; \boldsymbol{\xi})) p(\boldsymbol{\xi}) \mathrm{d} \xi_{i} \approx \int_{\Omega^{4}} s_{A}(V(t ; \boldsymbol{\xi})) p(\boldsymbol{\xi}) \mathrm{d} \xi_{i}=E\left[s_{0}\right]+\sum_{j=1}^{4} E\left[s_{j}\left(\xi_{j}\right)\right]
$$

where $E[f]$ is the expectation operator of $f$ with respect to the probability measure $p(\boldsymbol{\xi})$.

All four heterogeneous parameters here follow a uniform distribution: $I_{a p p}$ on $[17.5,32.5], V_{\text {syn }}$ on $[-1,1], V_{N a}$ on $[49,51]$, and $g_{N a}$ on $[2.55,3.05]$. The other parameters are given as follows:

$$
V_{N_{a}}=50, \quad V_{\text {syn }}=0, \quad g_{\text {syn }}=0.3, \quad g_{l}=2.4, \quad V_{l}=-65, \quad \varepsilon=0.1, \quad C=0.21 .
$$

The parameters for sparse grids and ANOVA are shown in Table 1. We also consider a direct Monte Carlo (MC) method with 10,000 points (i.e. 10,000 all to all coupled neurons) as a reference solution. Note that both sparse grids and ANOVA methods MC are non-intrusive methods, hence given the sampling (or collocation) points, we solve deterministic problems. Figure 1 shows the behavior of the $V_{i}$ and $h_{i}$ corresponding to 10 samples from the sparse grids in Table 1 .

TABLE 1. In sparse grids, the number of collocation points is determined by the level, i.e. the higher the level the more points. In the ANOVA method, $\mu$ is the number of collocation points in one direction and $\nu$ is the truncation dimension of the ANOVA decomposition, i.e. $\nu=2$ means that we consider only the first and second-order interaction terms. For these parameters 411 points are needed for the sparse grid method and 171 points for the ANOVA method.

\begin{tabular}{c|c|c} 
& Sparse Grid & ANOVA \\
\hline configuration & level $=3$ & $\mu=5, \nu=2$ \\
\hline number of points & 411 & 171
\end{tabular}

First we solve equations (7a) and (7b) for $V$ and $h$ using sparse grids, ANOVA and MC methods and compare the mean and variance of $V$ and $h$ derived from the three methods. For example, given sparse grids points and corresponding weights $\left\{\boldsymbol{\xi}^{(j)}, w^{(j)}\right\}_{j=1}^{N}$, the mean and variance for $V$ can be computed as

$$
\begin{aligned}
E[V](t) & =\sum_{j=1}^{N} V\left(t ; \boldsymbol{\xi}^{(j)}\right) w^{(j)} \\
\operatorname{Var}[V](t) & =\sum_{j=1}^{N} V^{2}\left(t ; \boldsymbol{\xi}^{(j)}\right) w^{(j)}-E[V]^{2}(t)
\end{aligned}
$$

where $V\left(t ; \boldsymbol{\xi}^{(j)}\right)$ is the solution to Equation (7a) with $\boldsymbol{\xi}=\boldsymbol{\xi}^{(j)}$. Figures 2 and 3 show the mean and variance for $V$ and $h$, respectively, calculated using the three methods, and the results agree well with one other. Note that they are visually indistinguishable but when zoomed in (inset figure), a 

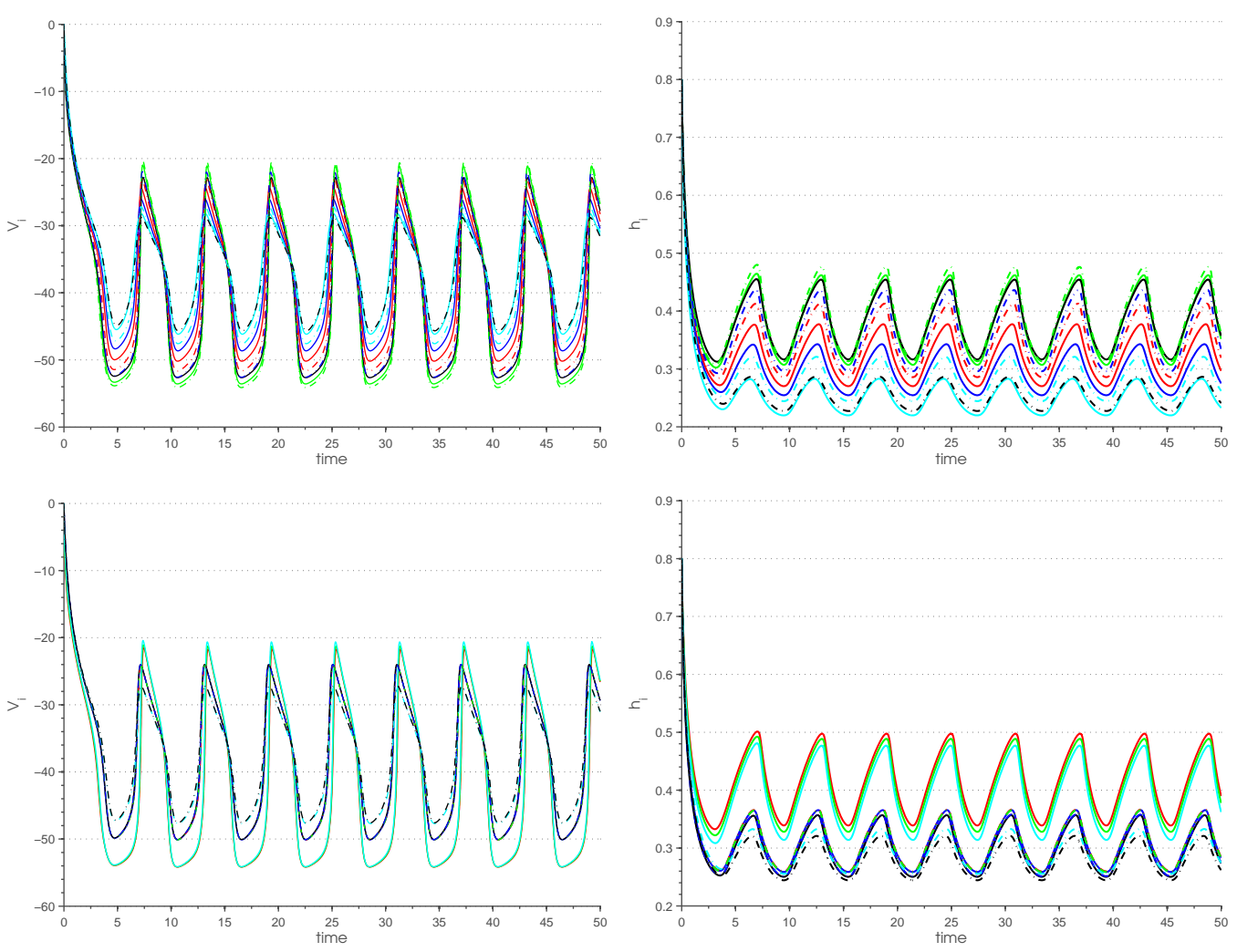

FIgURE 1. Solutions of Equations (1a) and (1b) when there are four heterogeneous parameters and samples come from sparse grids (Top) and ANOVA (bottom) with parameters given in Table 1, Left: $V_{i}$ as functions of time. Right: $h_{i}$ as functions of time. Different line colors correspond to different neurons (only ten neurons are shown) and they show that neurons with different parameters behave differently.

slight difference can be perceived between MC and the other two methods. This strongly suggests that the ANOVA method can help model high-dimensional heterogeneous parametric problems, in addition to its extensive use in high-dimensional uncertain parametric problems. Based on this observation, we consider to describe a low-dimensional system only using the ANOVA method from now on in this subsection.

Coarse Dynamics and Stability. We will now consider the gPC coefficients $\alpha_{i}$ and $\beta_{i}, i=$ $0,1, . . M$ for $V$ and $h$, respectively as our reduced, coarse-grained variables, i.e. we approximately 
represent $V$ and $h$ as

$$
\begin{aligned}
V(t ; \boldsymbol{\xi}) & =\sum_{i=0}^{M} \alpha_{i}(t) \Phi_{i}(\boldsymbol{\xi}) \\
h(t ; \boldsymbol{\xi}) & =\sum_{i=0}^{M} \beta_{i}(t) \Phi_{i}(\boldsymbol{\xi})
\end{aligned}
$$

where each $\Phi_{i}(\boldsymbol{\xi}), i=0, \ldots, M$ is a product of Legendre polynomials of the variables in $\boldsymbol{\xi}=$ $\left\{\xi_{1}, \xi_{2}, \xi_{3}, \xi_{4}\right\}$. We explore the long-term dynamics of (1) using these coarse-grained variables and compute gPC coefficients using ANOVA methods, as there are four heterogeneous parameters.
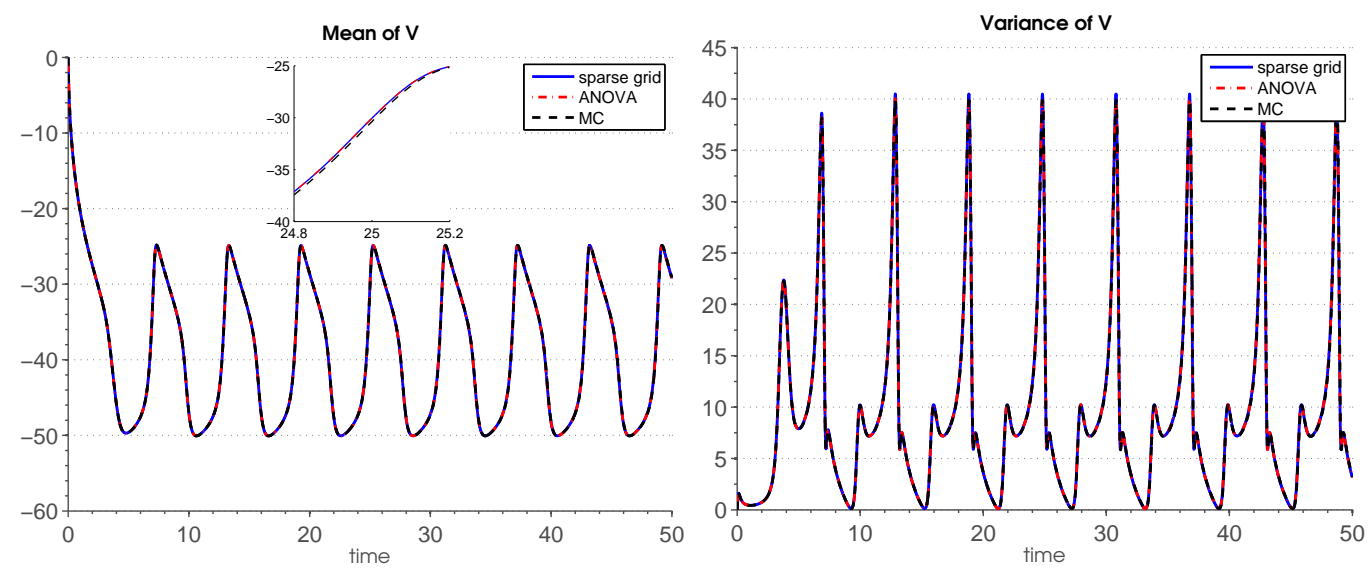

FIgURE 2. The mean (left) and variance (right) for $V$. MC simulations with 10,000 points are considered as the reference solution. Note that results from all methods are visually indistinguishable.

Equation-Free Computations. Availability of the governing equations for the variables of interest is a prerequisite to modeling and computation. However, if the underlying differential equations are nonlinear or nontrivial and $\boldsymbol{\xi}$ is high-dimensional, then the right hand side in Equation (12) is often coupled and very complicated making it almost impossible to obtain it in explicit, closed form. We circumvent this step using the equation-free (EF) framework for complex, multiscale systems modeling [14, 23. In this framework we can perform system-level computational tasks without explicit knowledge of the coarse-grained equations. This is accomplished through the operators that transform between coarse and fine variables. The mapping from coarse to fine variables is called the lifting operator $(L)$ while the mapping from fine to coarse variables is called the restriction operator $(R)$.

We denote the detailed (fine), microscopic time-evolution operator defined in Equation (7) by $\phi_{\tau}$ (where $\tau$ represents the number of time steps or iterations). The macroscopic evolution operator $\Phi_{\tau}$ can then be defined as follows:

$$
\Phi_{\tau}(\boldsymbol{\alpha}(t))=R \circ \phi_{\tau} \circ L(\boldsymbol{\alpha}(t))
$$


where $\boldsymbol{\alpha}(t)$ is the vector of $\mathrm{gPC}$ coefficients $\left(\alpha_{0}, \ldots, \alpha_{M}, \beta_{0}, \ldots, \beta_{M}\right)$ in Equation (27) representing the coarse-grained variables. The general procedure consists of five steps; (i) identifying observables that describe the coarse-grained variables $\boldsymbol{\alpha}$, (ii) constructing a lifting operator that maps the coarse variables to a fine scale realization, (iii) evolving the fine scale equations for certain amount of time, (iv) restricting the resulting fine variables to the coarse variables in order to estimate their time derivatives, and (v) repeating the procedure to perform specific computational tasks.

We first demonstrate coarse projective integration (CPI) [9]. The gPC coefficients $\alpha_{i}$ and $\beta_{i}, i=$ $0,1, . . M$ for $V$ and $h$ in Equation (27) are considered as the coarse-grained variables and obtained via Equation (10). For comparison, we also evolve the detailed (fine) coupled equation (7) from which we record the coefficients (coarse-grained variables) at every time step. The forward Euler method with a fixed step size of 0.001 is used as a time integrator. For CPI, the detailed (fine) coupled system (7) is integrated forward in time using short bursts of fine-scale simulations consisting of 7 steps. Then, the coarse variables $\boldsymbol{\alpha}$ are evaluated according to Equation (10) where the integral is computed by the ANOVA method given in Equation (22). The last few observations of the coarse variables $\boldsymbol{\alpha}$ are used to estimate their time-derivative. Finally we integrate the coarse variables with a forward Euler jump of 7 steps, thus save 7 inner integration steps at every 7 steps. Figure 4 shows the second and third gPC coefficients from coarse projective integration and from full detailed simulation, and shows that they agree well with each other. For the given parameters, in particular with $E\left[I_{a p p}\right]=25$, the network exhibits stable, synchronized periodic behavior as shown in Figure 5 .

The equation-free approach is also useful for computing long-time (stationary) states and their stability and dependence on parameters [14, 19]. The coarse time-stepper $\Phi_{\tau}(\boldsymbol{\alpha}(t))$ is defined as mapping from $\boldsymbol{\alpha}(t)$ to $\boldsymbol{\alpha}(t+\tau)$ via one iteration of the equation-free method as mentioned in the above: lifting a coarse-grained initial condition $\boldsymbol{\alpha}(t)$ to one or more consistent fine initial conditions, integrating the full (fine) model for a (short) time $\tau$, and then restricting to the coarse observable of the final fine state $\Phi_{\tau}$. In order to compute the stationary states we solve for the fixed point $\boldsymbol{\alpha}^{*}$ satisfying

$$
F_{\tau}(\boldsymbol{\alpha}) \equiv \Phi_{\tau}(\boldsymbol{\alpha})-\boldsymbol{\alpha}=0
$$

which is referred to as the coarse flow map. Iterative matrix-free linear algebra algorithms such as Newton-GMRES can be used to find zeros of such a function in the absence of explicit equations for the dynamics of the coarse variables $\boldsymbol{\alpha}$. Eigenvalues of the Jacobian of the coarse flow map $F_{\tau}$ evaluated at a fixed point reveal the (coarse grained) stability of that fixed point and help determine the nature of its potential bifurcations. Figure 6 shows the first 10 eigenvalues of the Jacobians of both the fine and coarse flow maps at equivalent fixed points. As the polynomial degree in the coarse flow map (the number of coarse variables) increases, these coarse eigenvalue estimates are expected to approach the leading eigenvalues of the Jacobian of the fine flow map, and this is clearly seen in Figure 6.

3.2. Case II: intrinsic and structural heterogeneity. We consider Equation (1) with the following physiological parameter values [17]

$$
V_{N_{a}}=50, \quad V_{s y n}=0, \quad g_{s y n}=0.3, \quad g_{l}=2.4, \quad V_{l}=-65, \quad \varepsilon=0.1, \quad C=0.21 .
$$

For a heterogeneous network, $I_{a p p}$ is chosen to follow a uniform distribution on [17.5,32.5], parameterized by $I_{a p p}=25+7.5 \omega$ where $\omega$ is uniformly distributed on $[-1,1]$. Neurons are connected in 
a Chung-Lu type network [16], i.e. neurons $i$ and $j$ are connected (i.e. $A_{i j}=1$ ) with probability

$$
p_{i j}=\min \left(\frac{\phi_{i} \phi_{j}}{\sum_{k} \phi_{k}}, 1\right)
$$

where $\phi_{i}=p N(i / N)^{r}, i=1, \ldots, N$ and $N$ is the number of neurons. We choose $N=512, p=0.5$ and $r=0.1$.

Clearly, we can consider the way the neurons are connected in the network as a different type of heterogeneity: a structural heterogeneity, where neurons are connected between them in different ways, as opposed to neurons having different individual parameters (an intrinsic heterogeneity, of the type we have discussing up to now). In our case, we assume that this structural heterogeneity is well described by the degree distribution: the degree of each neuron denoted by $\kappa$ is the important structural heterogeneous parameter, and its probability distribution is the degree distribution of our Chung-Lu network.

For these parameter values, and a particular realization of a Chung-Lu network with 512 neurons, we observe that the network eventually synchronizes, and all neurons evolve along a periodic trajectory (each in a slightly different periodic path, since the neurons differ both intrinsically and in their connectivities). At any point in time, the state at each neuron, $\left(V_{i}, h_{i}\right)(t)$ can be approximated by a smooth surface in two heterogeneous parameters $\boldsymbol{\xi}=\left(\kappa_{i}, I_{\mathrm{app}, i}\right)_{i=1}^{N}$ according to Equation (27). These are parameters in the sense that they do not change in time-they are still unique for each neuron. If indeed the behavior can be expressed as a function of our two heterogeneous parameters and time, this suggests that at every moment in time the values of the dynamic variables of each neuron would lie on a smooth surface, here a two dimensional one, parametrized by the two measures of heterogeneity. At every point in time the 512 individual variable values, one for every neuron, would lie on, or very close to, this surface.

Figure 7 shows the potential $V_{i}$ of all the neurons for $0 \leq t \leq 4$ and the evolving "heterogeneity surface" of the potential $V_{i}$ at two instances in time $t=1.27$ and 3.55 (marked on the figure) as a function of the two heterogeneous parameters, which are randomly picked at on a limit cycle. The fact that, for all practical purposes, the values of the variables for each neuron lie on, or close to such a smooth surface, implies that a $\mathrm{gPC}$ representation performs well as a coarse-grained descriptor of the heterogeneous neuronal population.

Figure 8 shows a phase portrait view of the limit cycle synchronized oscillation for all the neurons. In the insets we show, at seven different time instances, the potential V of each neurons (represented by colored filled circles), clearly lying on, or very close to, the smooth two-dimensional surface of the coarse-grained description.

\section{Conclusion}

We have proposed and demonstrated the use of several distinct forms of dimension reduction for the computationally efficient study of heterogeneous networks of coupled neurons. In Case I we considered an all-to-all coupled network with four independent heterogeneous parameters. To efficiently simulate such a network we need to approximate a four-dimensional integral, which we accomplished using ANOVA methods. A reduced model of this type of network can also be formulated using coefficients in a polynomial chaos expansion in the heterogeneous parameters as the "coarse" variables. Having such a reduced model leads to an improvement in the speed for a variety of computations of interest (direct simulation, coarse limit cycle computation, coarse stability analysis) which we demonstrated using the equation-free framework. 
In Case II we considered a network with both intrinsic and structural heterogeneity, and showed that we could expand the state variables in polynomials of both the intrinsically varying parameter and a feature of the network connectivity - in this case, the degree of each neuron. To do this, we need to construct orthogonal polynomials with respect to the network degree distribution. If this (integer) distribution is known a priori, then the polynomials can be found in the literature [25], or easily constructed using the recurrence relation [24]. If the distribution is unknown, and we only have samples of it available, then the convergence of the "empirical" polynomials based on the sampled distributions, to the "true" distributions at the limit of infinite neurons becomes an interesting research problem that we are currently investigating. We believe that all these approaches can play an important practical role in accelerating the computational study (and, in general, the modeling) of complex heterogeneous networks, and we are exploring the practical limits of (a) how many independently distributed heterogeneous parameters one can usefully approximate and (b) the modeling of heterogeneities that are not independently distributed, but rather exhibit correlations.

Acknowledgements. This work was partially supported by the US National Science Foundation and by the US AFOSR. The hospitality and support of the Institute for Advanced Study at the T. U. Muenchen, where I.G.K was a Hans Fischer Senior Fellow, and C.L. a visitor is gratefully acknowledged.

\section{REFERENCES}

[1] Peter Ashwin and James W Swift. The dynamics of $n$ weakly coupled identical oscillators. Journal of Nonlinear Science, 2(1):69-108, 1992.

[2] Katherine A Bold, Yu Zou, Ioannis G Kevrekidis, and Michael A Henson. An equation-free approach to analyzing heterogeneous cell population dynamics. Journal of mathematical biology, 55(3):331-352, 2007.

[3] Robert J Butera, John Rinzel, and Jeffrey C Smith. Models of respiratory rhythm generation in the pre-bötzinger complex. i. bursting pacemaker neurons. Journal of neurophysiology, 82(1):382-397, 1999.

[4] Robert J Butera, John Rinzel, and Jeffrey C Smith. Models of respiratory rhythm generation in the pre-bötzinger complex. ii. populations of coupled pacemaker neurons. Journal of Neurophysiology, 82(1):398-415, 1999.

[5] Justin R Dunmyre and Jonathan E Rubin. Optimal intrinsic dynamics for bursting in a three-cell network. SIAM Journal on Applied Dynamical Systems, 9(1):154-187, 2010.

[6] R. Fisher. Statistical Methods for Research Workers. Oliver and Boyd, 1925.

[7] Jasmine Foo, Xiaoliang Wan, and George Em Karniadakis. The multi-element probabilistic collocation method (me-pcm): Error analysis and applications. Journal of Computational Physics, 227(22):9572-9595, 2008.

[8] Jasmine Y. Foo and George Em Karniadakis. Multi-element probabilistic collocation in high dimensions. Journal of Computational Physics, 229:1536-1557, 2010.

[9] C William Gear and Ioannis G Kevrekidis. Projective methods for stiff differential equations: problems with gaps in their eigenvalue spectrum. SIAM Journal on Scientific Computing, 24(4):1091-1106, 2003.

[10] Thomas Gerstner and Michael Griebel. Numerical integration using sparse grids. Numerical algorithms, 18(34):209-232, 1998.

[11] Roger G Ghanem and Pol D Spanos. Stochastic finite elements: a spectral approach. Courier Corporation, 2003.

[12] Brian Hassard. Bifurcation of periodic solutions of the hodgkin-huxley model for the squid giant axon. Journal of Theoretical Biology, 71(3):401-420, 1978.

[13] W. Hoeffding. A class of statistics with asymptotically normal distributions. Annals of Math. Statist., 19:293$325,1948$.

[14] Ioannis G Kevrekidis, C William Gear, James M Hyman, Panagiotis G Kevrekidis, Olof Runborg, Constantinos Theodoropoulos, et al. Equation-free, coarse-grained multiscale computation: Enabling mocroscopic simulators to perform system-level analysis. Communications in Mathematical Sciences, 1(4):715-762, 2003.

[15] Carlo R Laing and Ioannis G Kevrekidis. Periodically-forced finite networks of heterogeneous globally-coupled oscillators: a low-dimensional approach. Physica D: Nonlinear Phenomena, 237(2):207-215, 2008.

[16] Carlo R Laing, Karthikeyan Rajendran, and Ioannis G Kevrekidis. Chimeras in random non-complete networks of phase oscillators. Chaos: An Interdisciplinary Journal of Nonlinear Science, 22(1):013132, 2012. 
[17] Carlo R Laing, Yu Zou, Ben Smith, and Ioannis G Kevrekidis. Managing heterogeneity in the study of neural oscillator dynamics. Journal of mathematical neuroscience, 2(1):5, 2012.

[18] Sung Joon Moon, Katherine A Cook, Karthikeyan Rajendran, Ioannis G Kevrekidis, Jaime Cisternas, and Carlo R Laing. Coarse-grained clustering dynamics of heterogeneously coupled neurons. The Journal of Mathematical Neuroscience (JMN), 5(1):1-20, 2015.

[19] Sung Joon Moon, R. Ghanem, and I. G. Kevrekidis. Coarse graining the dynamics of coupled oscillators. Phys. Rev. Lett., 96:144101, Apr 2006.

[20] Jonathan Rubin and David Terman. Synchronized activity and loss of synchrony among heterogeneous conditional oscillators. SIAM Journal on Applied Dynamical Systems, 1(1):146-174, 2002.

[21] Jonathan E Rubin. Bursting induced by excitatory synaptic coupling in nonidentical conditional relaxation oscillators or square-wave bursters. Physical Review E, 74(2):021917, 2006.

[22] I.M. Sobol'. Global sensitivity indices for nonlinear mathematical models and their monte carlo estimates. Mathematics and Computers in Simulation, 55:271-280, 2001.

[23] Constantinos Theodoropoulos, Yue-Hong Qian, and Ioannis G Kevrekidis. coarse stability and bifurcation analysis using time-steppers: A reaction-diffusion example. Proceedings of the National Academy of Sciences, 97(18):9840-9843, 2000.

[24] Xiaoliang Wan and George Em Karniadakis. Beyond wiener-askey expansions: handling arbitrary pdfs. Journal of Scientific Computing, 27(1-3):455-464, 2006.

[25] Dongbin Xiu. Numerical methods for stochastic computations: a spectral method approach. Princeton University Press, 2010.

[26] Dongbin Xiu and Jan S Hesthaven. High-order collocation methods for differential equations with random inputs. SIAM Journal on Scientific Computing, 27(3):1118-1139, 2005.

[27] Dongbin Xiu and George Em Karniadakis. The wiener-askey polynomial chaos for stochastic differential equations. SIAM journal on scientific computing, 24(2):619-644, 2002.

[28] Xiu Yang, Minseok Choi, Guang Lin, and George Em Karniadakis. Adaptive anova decomposition of stochastic incompressible and compressible flows. Journal of Computational Physics, 231(4):1587-1614, 2012.

(Minseok Choi and Tom Bertalan) Department of Chemical and Biological Engineering, Princeton UniVersity, Princeton, NJ 08544, USA

(Carlo R. Laing) Institute of Natural and Mathematical Sciences, Massey University, Auckland, New ZEALAND

(Ioannis G Kevrekidis) Department of Chemical and Biological Engineering and Program in Applied and Computational Mathematics, Princeton University, Princeton, NJ 08544, USA 

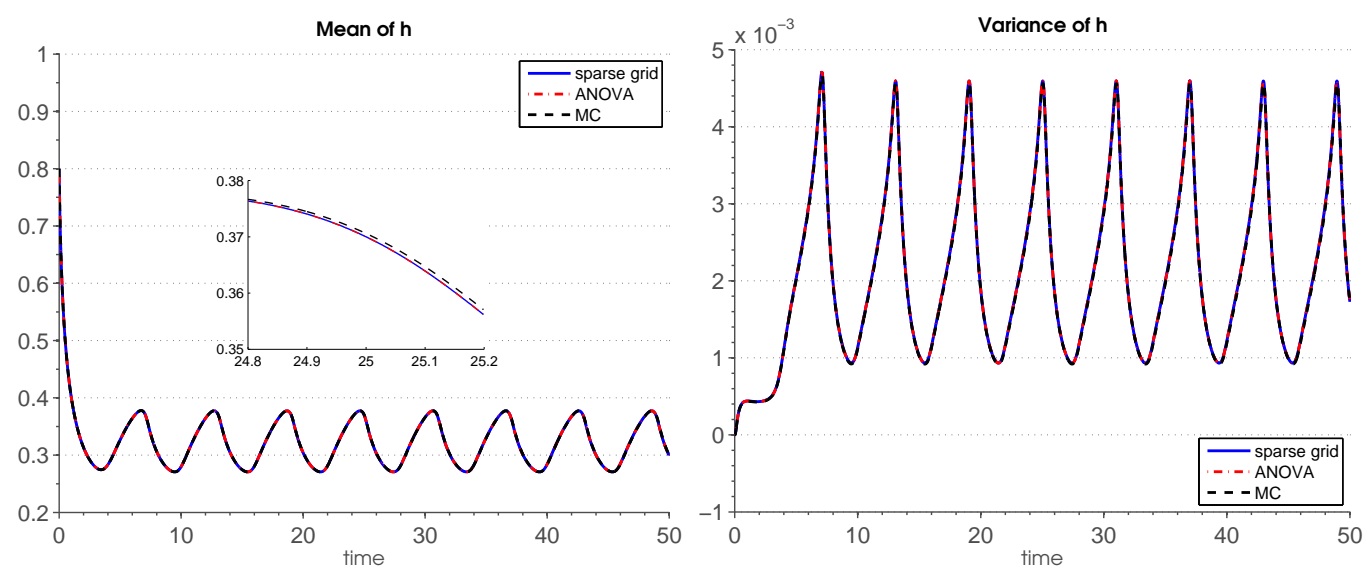

FiguRE 3. The mean (left) and variance (right) for $h$. MC simulations with 10,000 points are considered as the reference solution. Note that all methods are visually indistinguishable.
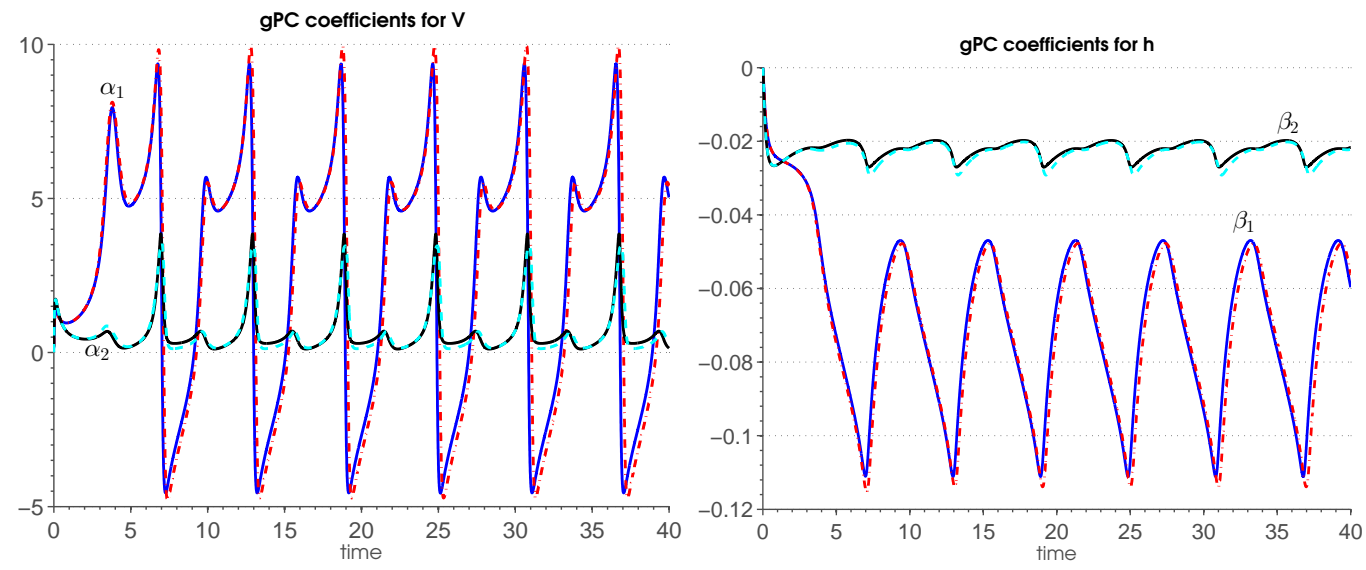

Figure 4. Coarse projective integration (dashed lines) and detailed (fine) coupled dynamics (solid lines) for $V$ (left) and $h$ (right). Two PC coefficients $\left(\alpha_{1}, \alpha_{2}\right)$ and $\left(\beta_{1}, \beta_{2}\right)$ are shown for $V$ and $h$, respectively. Forward Euler with a fixed step size of 0.001 is used as a time integrator. For coarse projective integration, it jumps with a forward Euler of 7 step after estimating time derivatives. 

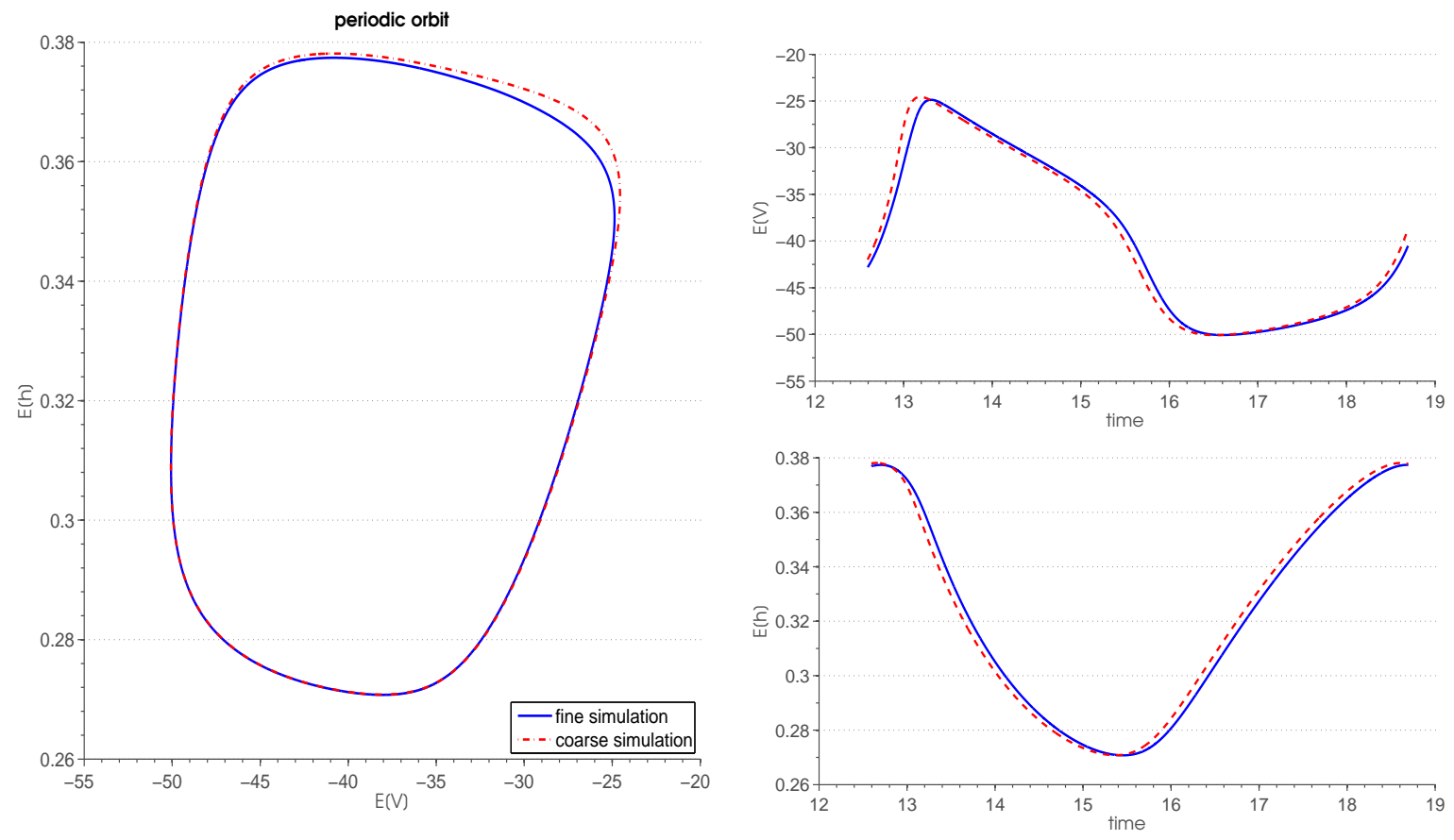

Figure 5. Left: Periodic orbit of the mean of $V$ and the mean of $h$ when $E\left[I_{a p p}\right]=25$. Coarse projective integration (dashed) and detailed (solid) simulation. Temporal profile of $E[V]$ (top right) and $E[h]$ (bottom right) corresponding to one period of limit cycle. 


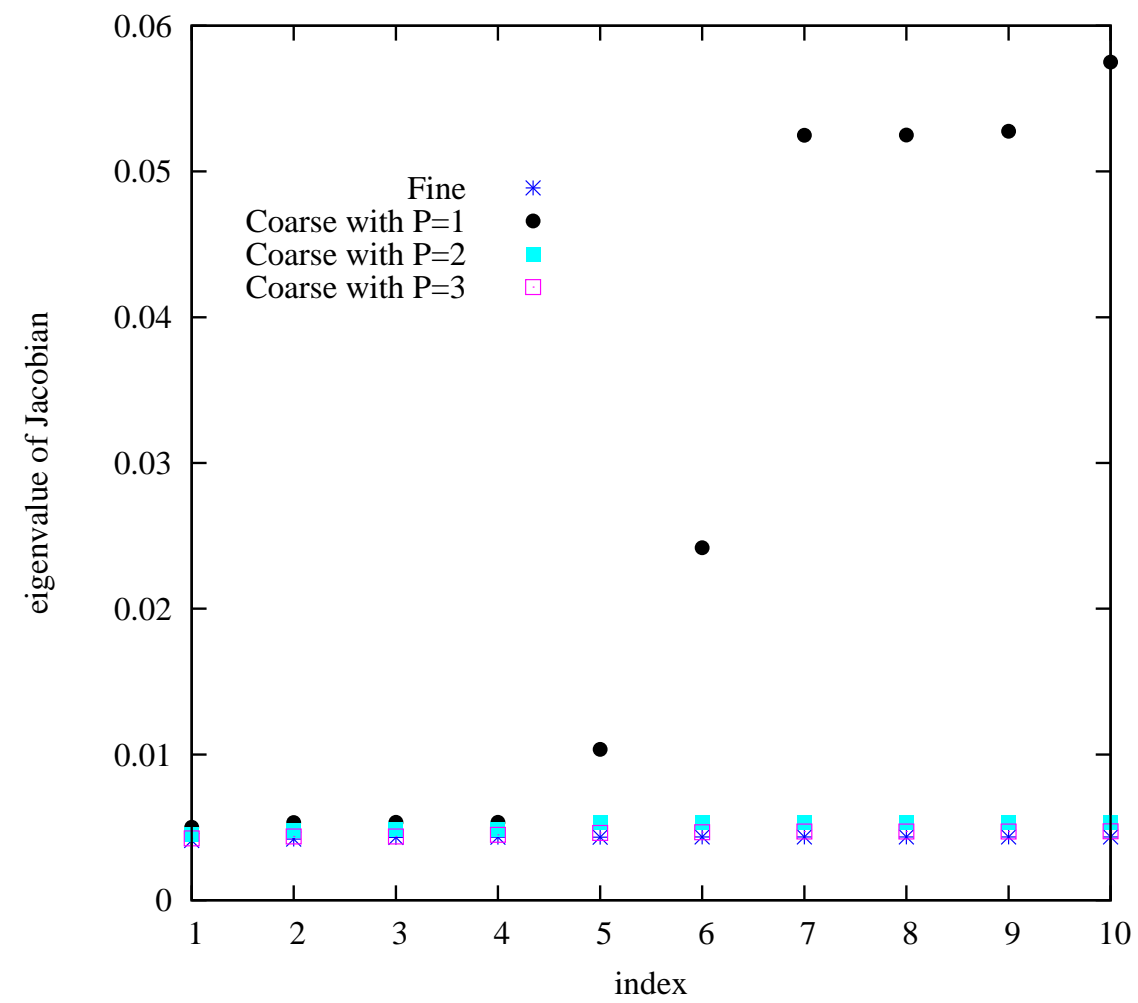

FIGURE 6. Eigenvalues of the Jacobian of the fine flow map and the coarse flow map at corresponging fixed points, obtained with three different gPC orders: $P=1$ (leading to 10 coefficients), $P=2$ (30 coefficients), and $P=3$ (70 coefficients). As the polynomial degree -and thus the number of coarse variables- increases, the eigenvalues from the coarse flow simulation show increasingly better agreement with those from the fine simulation. 

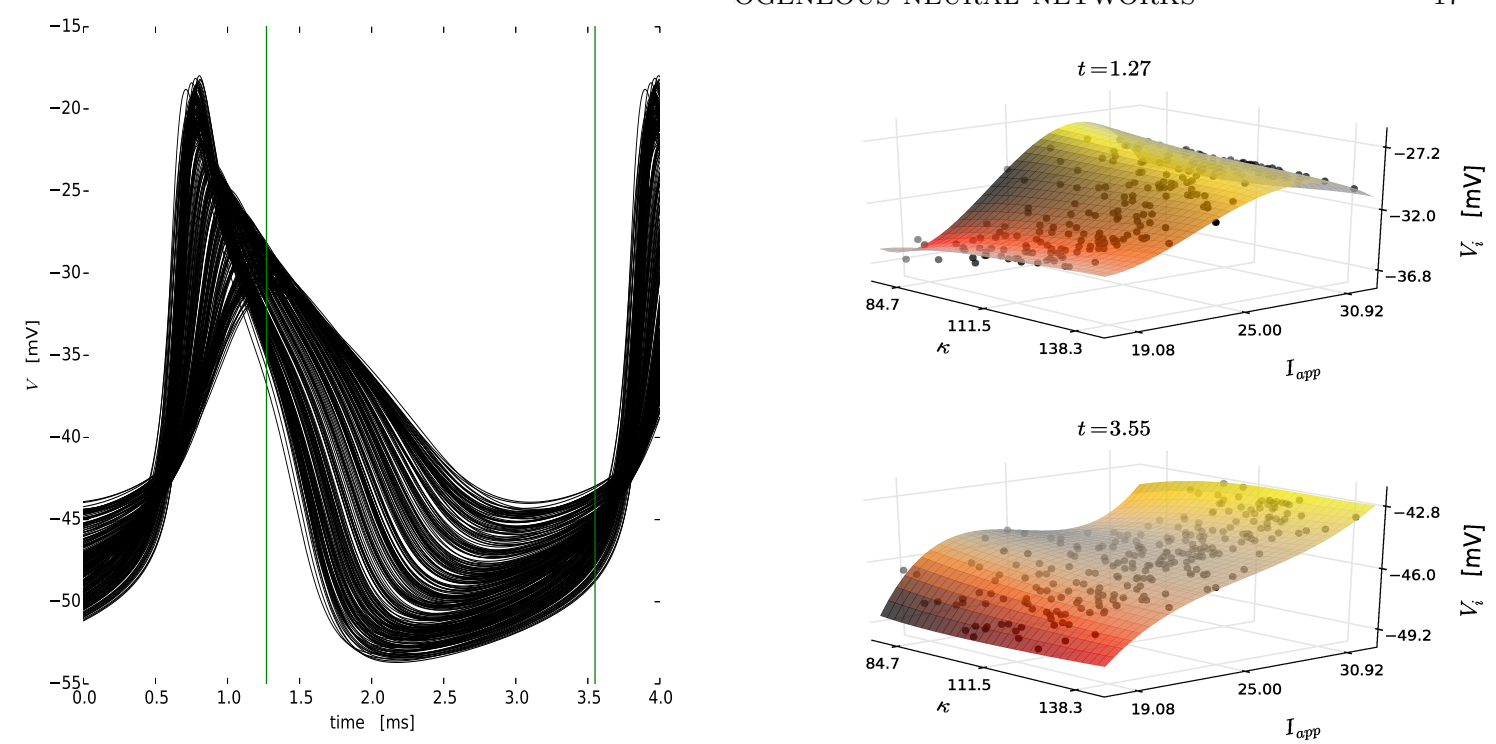

Figure 7. Left: Potential $\left(V_{i}\right)$ in Equation (1a) with two heterogeneous parameters $\left(\kappa_{i}, I_{\mathrm{app}, i}\right)$. Surface of the $V_{i}$ as a function of the two parameters $\left(\kappa_{i}, I_{\mathrm{app}, i}\right)$ at $t=1.27$ (top right) and $t=3.55$ (bottom right). The neurons lie on, or very close to, the smooth manifold. Initial conditions for the integration of (1a) at $t=0$ are a point $\vec{X}(t=0 ; \boldsymbol{\xi})=\{\vec{V}, \vec{h}\}\left(t=0 ;\left\{\kappa, I_{a p p}\right\}\right)$ found by simple forward integration to be on or very close to the attracting limit cycle. In the left panel, we show approximately one period of this limit cycle, observed in the $512 \mathrm{~V}$ traces over time. 


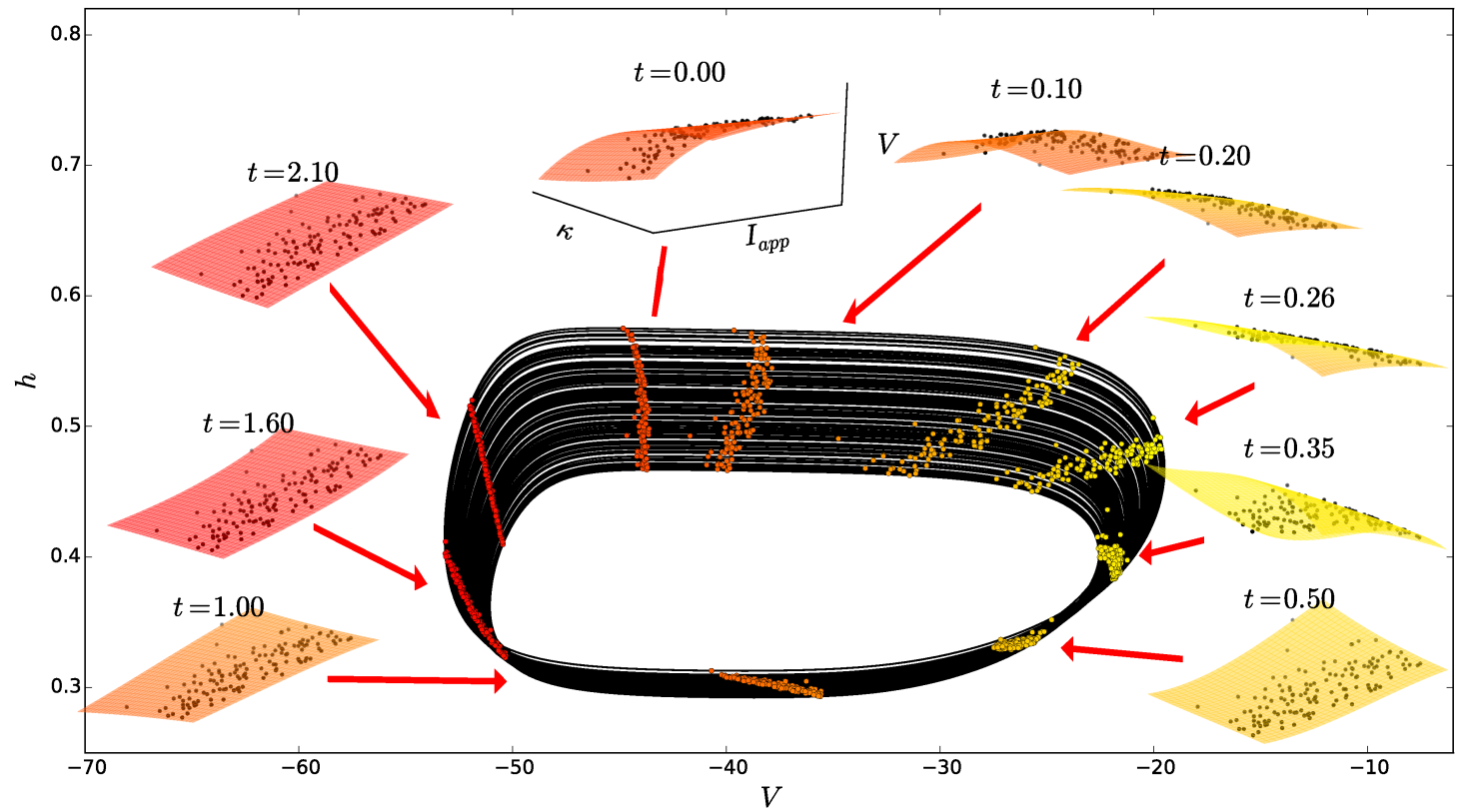

Figure 8. Limit cycles of the $\left(V_{i}, h_{i}\right)$ for $N=512$ neurons. Each filled circle represents the potential of one neuron, with different colors denoting different time snapshots along the synchronized oscillation. The oscillations proceed in the clockwise direction, and the surfaces in the insets show the $V_{i}$ as functions of the two heterogeneous parameters at nine different times cut. 